\title{
Use of the maximal ST/HR slope to estimate myocardial ischaemia after recent myocardial infarction
}

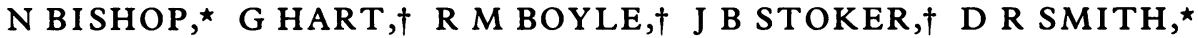 \\ D A S G MARY* \\ From the ${ }^{\star}$ Department of Cardiovascular Studies, University of Leeds, and $†$ Killingbeck Hospital, Leeds
}

SUMMARY Fifty two patients were examined 4-6 weeks after myocardial infarction to assess whether factors other than coronary artery narrowing affect the maximal ST/HR slope which is used as an index of myocardial ischaemia. The slope was compared with indices of myocardial scarring or cardiac enlargement derived from $x$ ray and echocardiographic and angiocardiographic investigations. In $35(67 \%)$ patients the slope failed to predict the severity of myocardial ischaemia attributable to coronary artery narrowing: in $14(27 \%)$ patients the slope overestimated the findings of coronary angiography and in $21(40 \%)$ patients the slope underestimated the findings of coronary angiography. In the remaining $17(33 \%)$ patients the slope accorded with the assessment of myocardial ischaemia by coronary angiography. Underestimation by the slope was associated with significantly poorer left ventricular function and a lower ejection fraction, indicating a greater degree of myocardial scarring.

To assess whether overestimation was related to cardiac enlargement with better preservation of ventricular function a follow up examination was performed six months after infarction. In the overestimated group 11 patients were followed up and seven of them showed a reduction in the maximal ST/HR slope which correlated with a reduction in the cardiothoracic ratio. This suggested that cardiac enlargement had contributed to myocardial ischaemia.

The results suggest that if the maximal ST/HR slope is an index of exercise induced myocardial ischaemia after recent infarction, it is subject to the influences of coronary artery narrowing as well as those of scarring and cardiac enlargement.

Studies in Leeds have shown that the maximal $\mathrm{ST} / \mathrm{HR}$ slope is an index of myocardial ischaemia when related to coronary artery narrowing in selected populations of patients with angina, ${ }^{12}$ and that the slope is influenced by cardiac enlargementfor example in a selected population of patients with aortic valve disease and normal coronary arteriograms. ${ }^{3}$

In patients who have had an acute myocardial infarction exercise induced myocardial ischaemia has been related to coronary artery stenosis, though other factors, such as cardiac enlargement or the

Requests for reprints to Dr D A S G Mary, Department of Cardiovascular Studies, University of Leeds, Leeds LS2 9JT.

Accepted for publication 1 December 1986 extent of myocardial scarring and muscle tissue loss, could be argued to affect the ischaemic process. ${ }^{4-6}$ We report a comparison of the maximal ST/HR slope and the results of angiocardiography in patients with recent myocardial infarction as a further test of the reliability of the slope as an index of myocardial ischaemia.

\section{Patients and methods}

Consecutive patients were prospectively studied 4-6 weeks after myocardial infarction. The diagnosis of myocardial infarction was confirmed by the presence of at least two of the following criteria (a) typical history of chest pain lasting at least 30 minutes; $(b)$ characteristic serial electrocardiographic changes, that is ST segment elevation, $T$ wave inversion, and 
the appearance of new $Q$ waves; $(c)$ a rise in serum creatine kinase to at least twice the upper limit of normal. Patients with complications after infarction-for example cardiogenic shock, cardiac failure, arrhythmias, or conduction disturbances occurring 72 hours after infarction; acute haemodynamic complications-for example mitral regurgitation, pulmonary embolism; and unstable angina were not considered for study. Patients with uncontrolled hypertension, concomitant valve or chronic cardiac disease, or other medical conditions making exercise difficult were excluded from study. No patient on digoxin was included in the study.

All patients gave informed consent to the investigations and the study was approved by the local ethics committee.

We examined the effects of myocardial scarring and cardiac enlargement on myocardial ischaemia early after infarction as assessed by the maximal ST/HR slope. Wall scarring was assessed by angiographic patterns of left ventricular wall motion ${ }^{7}$ and ejection fractions. To assess the influence of cardiac enlargement patients were re-examined six months after infarction and individual changes in heart size were measured, with the patients acting as their own controls. Changes in cardiothoracic ratio were measured on standard chest $x$ rays $^{89}$ and ventricular dimensions were obtained by cross sectional echocardiography. ${ }^{10} \mathrm{~A}$ blind comparison was made between the maximal ST/HR slope and the results of angiocardiography, chest $x$ rays, and echocardiography.

\section{EXERCISE TEST}

In each patient the exercise test was performed 4-6 weeks after a confirmed myocardial infarction. The details of the exercise test have been reported elsewhere. ${ }^{11}$ Briefly, the exercise test was performed on an electrically braked bicycle ergometer with the patient in the upright position. Each test was preceded by a short preliminary exercise to determine the stepped increases in workload necessary to obtain stepped increases in heart rate of approximately 10 beats/minute, to enable adequate recordings by electrode replacement if required, and to familiarise the patient with the procedure. During the test the workload was increased every three minutes, with the test being terminated for the following reasons: anginal pain, ST segment depression $>0.3 \mathrm{mV}$, more than six multifocal ectopic impulses per minute or tachycardia, a decrease in blood pressure, or exhaustion.

The electrocardiogram was recorded from 13 leads-12 conventional leads and a bipolar lead (CM5) - with an ink jet Mingograph recorder (Siemens type 62 ). The leads were calibrated by setting the amplifier gain so that a $1 \mathrm{mV}$ signal caused a displacement of $10 \mathrm{~mm}$. Records from the 13 electrocardiographic leads were obtained at rest, during the last minute of each exercise step, and for at least five minutes after the end of exercise. The electrocardiogram was monitored throughout the test and recovery period. Systemic blood pressure was measured before, during the third minute of each exercise step, and during the recovery period.

The ST segment level was measured at a point $80 \mathrm{~ms}$ after the end of the QRS complex; a line drawn between consecutive PR segments was taken as the zero reference. This measurement was made with a magnifying glass fitted with a graticule marked in $0.1 \mathrm{~mm}$ divisions. The heart rate (beats/minute) was calculated by measuring the $\mathrm{R}-\mathrm{R}$ interval on the electrocardiogram.

The measurements of ST segment level and heart rate were made from electrocardiographic records obtained during the steady state (heart rate value within $\pm 5 \%$ of the mean) attained during the third minute of each exercise step. The values of heart rate and ST segment level were taken as the average value measured in at least 10 consecutive cardiac cycles obtained from records of all 13 electrocardiographic leads.

The maximal ST/HR slope was derived by performing regression analysis on the relation between the ST segment level and heart rate in each of the 13 electrocardiographic leads. In each lead the steepest slope of the computed regression lines which showed a linear regression was noted. The maximal ST/HR slope of all the 13 electrocardiographic leads was used as an index of myocardial ischaemia and this has units of mm per beat minute per $1000 .{ }^{111}$

\section{ANGIOCARDIOGRAPHY}

In each patient within one week of the exercise test selective coronary arteriography and left ventriculography were performed and reported on by the cardiologists without knowledge of the results of the exercise test.

Significant coronary artery disease was defined as luminal narrowing of the diameter of $>75 \%$ in any of the three major coronary arteries. ${ }^{12} \mathrm{We}$ used the same method of scoring angiograms so that we could compare the results of our study with previous studies in patients with angina. ${ }^{11} 12$ Percentage narrowing was assessed by a visual impression based on views of the vessels in more than one plane. The degree of stenosis of a long lesion was estimated at its narrowest point and in vessels that contained more than one lesion the degree of stenosis was that of the most severe lesion. Patients were divided into four groups: those with no significant coronary artery disease and these with single, double, and triple ves- 


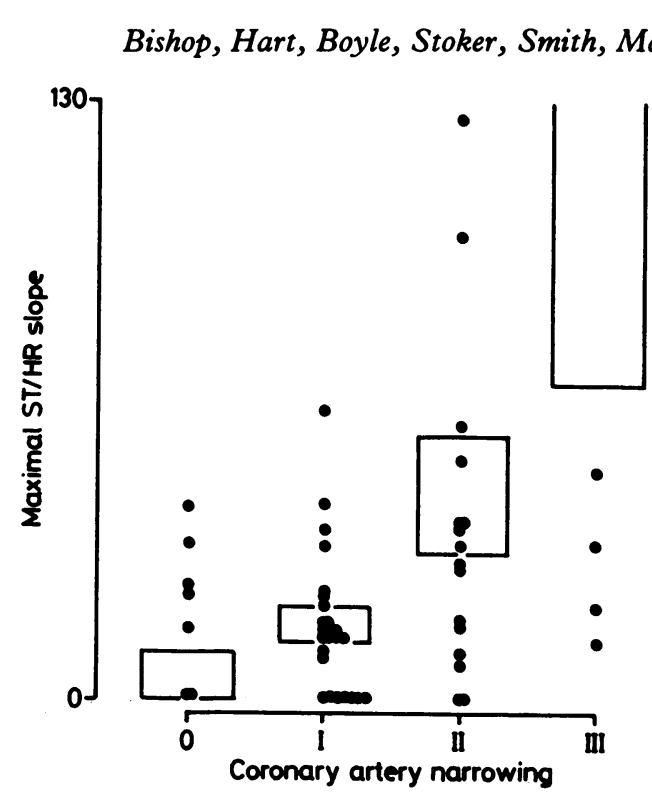

Fig 1 Comparison of individual maximal $S T / H R$ slope values ( $\mathrm{mm}$ per beat min per 1000) with independent assessment of myocardial ischaemia from coronary angiography. The boxed areas indicate the equivalent slope values for that degree of coronary artery narrowing ( $0=$ no significant stenosis, $I=$ single vessel, $I I=$ double vessel, and $I I I=$ triple vessel disease) previously found in a selected population with angina. ${ }^{1}$

\section{Results}

CLINICAL DETAILS

Fifty three consecutive patients prospectively underwent exercise testing 24-44 (mean 34.7) days after a confirmed myocardial infarction. One of these patients was excluded from the study because we could not obtain an electrocardiogram that was adequate for the derivation of the maximal ST/HR slope. Blind comparison of the value of the maximal ST/HR slope and the findings of angiocardiography was made in 52 patients (six women, 46 men; aged 37-64 (52.6) years).

Twenty eight patients suffered an inferior myocardial infarction and in the remaining 24 patients there was an anterior infarction. Eight patients gave a history of previous myocardial infarction. At the time of examination the following drugs were being taken: $\beta$ blocker (18 patients), thiazide diuretic (13 patients), frusemide ( 2 patients), nitrates (6 patients), nifedipine (2 patients), and methyldopa ( 1 patient). Twenty patients were receiving no drug treatment. In most patients followed up at six months there had been no change in drug treatment. Three patients were on additional vasodilator treatment (nifedipine or nitrates) which may increase the slope. ${ }^{1516}$ 


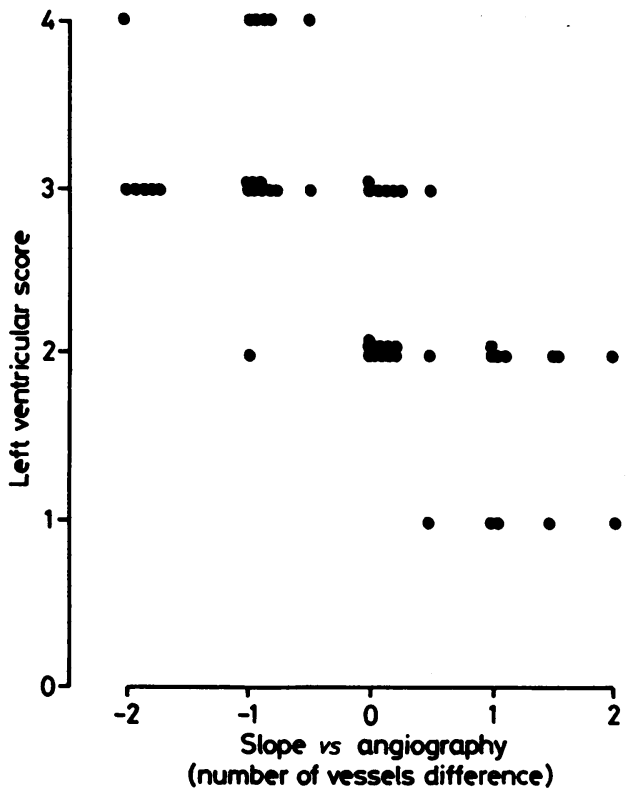

Fig 2 Individual discrepancies between the assessment of myocardial ischaemia by the maximal $S T / H R$ slope and by coronary angiography (in terms of the number of vessels difference) ( $\mathrm{mm}$ per beat min per 1000) related to left ventricular score.

For instance if the slope suggested single vessel disease and angiography showed double vessel disease, this would give a difference of -1 . If angiography demonstrated single vessel disease and the slope value suggested double vessel disease, this would give a difference of +1 . Where the slope gave intermediate values, say between single vessel and double vessel disease, this is taken as 1.5 for the purpose of comparison with angiography.

COMPARISON BETWEEN THE VALUE OF THE MAXIMAL ST/HR SLOPE AND THE ANGIOGRAPHIC EXTENT OF CORONARY ARTERY NARROWING

The maximal ST/HR slope increased as the severity of ischaemia worsened (fig 1). Eight patients had values of the slope in between the previously described discrete categories for no significant disease, single, double, and triple vessel disease. There was agreement between coronary angiographic assessment of the severity of myocardial ischaemia and the slope in only $17(33 \%)$ of the 52 patients. In the remaining $35(67 \%)$ patients the slope failed to predict the severity of myocardial ischaemia; the slope either underestimated the angiographic extent of significant coronary artery narrowing (21 patients $(40 \%))$ or it overestimated the angiographic extent of coronary artery narrowing (14 patients (27\%)). No patient with significant narrowing of all three coronary arteries had a maximal ST/HR slope equivalent to triple vessel disease.

Because the underestimation of the extent of coronary artery disease could be due to the effect of myocardial scarring, ${ }^{6}$ we examined indices of left ventricular function (the left ventricular score and ejection fraction).

\section{LEFT VENTRICULAR FUNCTION}

Left ventricular score

For patients given a left ventricular score of 4, indicating severe left ventricular dilatation and severe impairment of wall mobility, the value of the slope consistently underestimated the extent of coronary artery narrowing (fig 2). While for those patients judged to have the best preserved left ventricular function-that is with a left ventricular score of 1 -the slope consistently overestimated the extent of coronary artery narrowing (fig 2). There is a tendency for a discrepancy between the slope and angiography to be more likely if the left ventricular score is high; linear regression analysis showed that this trend is significant $(r=-0.68, \mathrm{p}<0.001)$ (fig 2).

A higher left ventricular score is significantly associated with larger end systolic and larger end diastolic volumes, and a lower ejection fraction (fig 3).

\section{Left ventricular volumes}

Underestimation by the maximal ST/HR slope of the severity of coronary artery narrowing is associated with larger end systolic and end diastolic volumes (table). Similar results were obtained when we used left ventricular areas derived from single plane angiography.

\section{Ejection fraction}

Underestimation by the maximal ST/HR slope is associated with a lower ejection fraction (fig 4; $\mathrm{r}=$ $0.60, \mathrm{p}<0.001$ ).

In patients with single vessel disease there is a significant tendency between the value of the maximal ST/HR slope and the left ventricular score $(r=-0.63, p<0.05)$, lower values of the slope being associated with higher scores (fig 5). A trend is seen when the value of the slope is related to the ejection fraction $(r=+0.57, p<0.05)$.

FOLLOW UP STUDY

Of the total of 52 patients, 44 were re-examined six months after infarction; three had died, one had an embolic stroke, one had undergone coronary artery surgery, one had required permanent pacing for sinuatrial disease, and two patients declined further exercise tests.

All three patients who died had poor left ventricu- 
lar function (left ventricular scores of 3, 4, and 4; ejection fractions of 53,27 , and $29 \%$ respectively) and in all three the slope underestimated the severity of coronary artery narrowing.

To assess the influence of cardiac enlargement

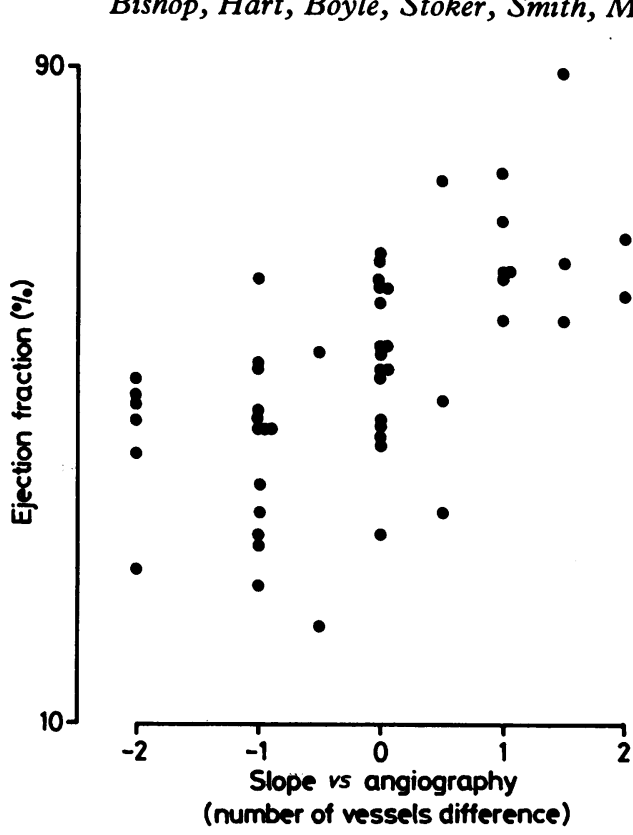

Fig 4 Individual discrepancy between assessment of myocardial ischaemia by the maximal ST/HR slope ( $\mathrm{mm}$ per beat min per 1000) and by coronary angiography (in terms of the number of vessels difference) in relation to ejection fraction.

early after myocardial infarction we re-examined patients at six months and examined individual changes in heart size in relation to individual changes in the severity of myocardial ischaemia as assessed by the maximal ST/HR slope. In the 44 patients re-examined at six months there was a statistically significant reduction in cardiothoracic ratio $(p<0.02)$ from an initial mean (SD) value of 48.5 $(4 \cdot 5) \%$. The reductions in heart size as assessed by the cardiothoracic ratio were greater in the overestimate group, though this difference from the agreement and underestimate groups is not statistically significant.

Table Ejection fraction and left ventricular volumes (mean (SD) (range)) for the three groups of patients in whom the maximal ST/HR slope showed either overestimation or underestimation or agreement with the findings of coronary angiography

\begin{tabular}{lccc}
\hline & $\begin{array}{c}\text { Overestimation } \\
(n=14)\end{array}$ & $\begin{array}{c}\text { Agreement } \\
(n=17)\end{array}$ & $\begin{array}{l}\text { Underestimation } \\
(n=21)\end{array}$ \\
\hline Ejection fraction $(\%)$ & $64 \cdot 8(12 \cdot 6)$ & $54 \cdot 4(9 \cdot 3) \star$ & $43 \cdot 7(10 \cdot 5) \dagger \ddagger$ \\
End diastolic volume $(\mathrm{ml})$ & $(36-89)$ & $(33-67)$ & $(22-64)$ \\
End systolic volume $(\mathrm{ml})$ & $(07 \cdot 4(28 \cdot 2)$ & $107.5(29)$ & $(75-165)$ \\
& $(72-146)$ & $(44 \cdot 4) \S$ \\
& $(10-68)$ & $(39-230)$ & $(20 \cdot 2)$ \\
\hline
\end{tabular}

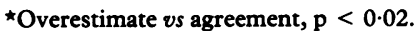

† Overestimate $v s$ underestimate, $\mathrm{p}<0.001$

tAgreement vs underestimate, $p<0.01$.

$\$$ Agreement or overestimate vs underestimate, $\mathrm{p}<0.02$ 


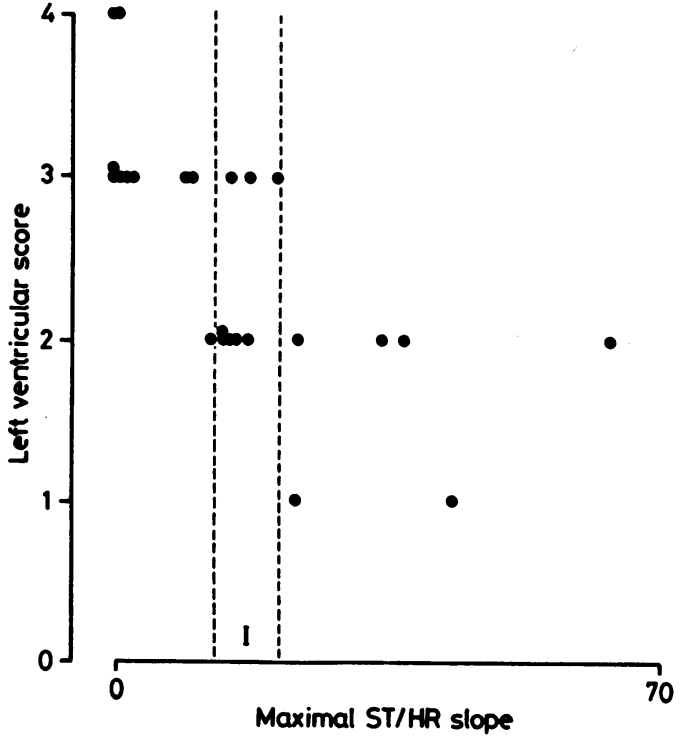

Fig 5 Individual values of the maximal ST/HR slope $(\mathrm{mm}$ per beat min per 1000) related to left ventricular score for the 25 patients with single vessel disease. The range of the slope for single vessel $(I)$ disease as found in selected patients with angina ${ }^{1}$ is shown by the broken lines.

Eleven of the 14 patients in whom the slope had overestimated the extent of coronary artery narrowing were re-examined at six months. Eight of these 11 patients showed a reduction in heart size as assessed by the cardiothoracic ratio. Seven of these eight patients had an associated reduction in the maximal ST/HR slope (fig 6). In contrast, such a

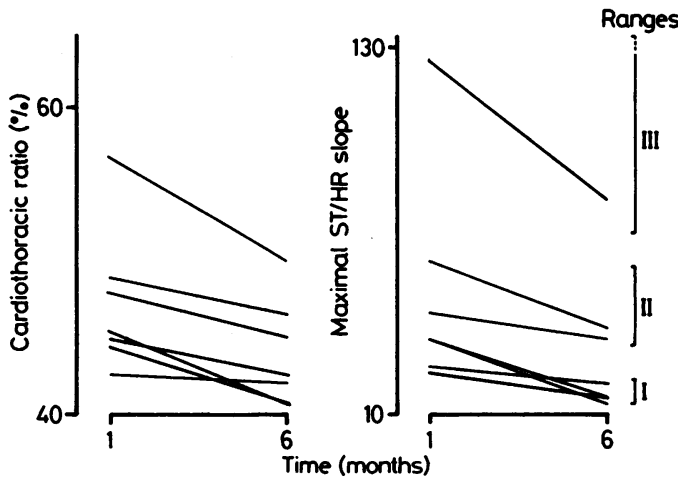

Fig 6 Individual changes in cardiothoracic ratio and maximal ST/HR slope ( $\mathrm{mm}$ per beat min per 1000) for seven patients from the group in which the slope overestimated the findings of coronary angiography. The ranges of the maximal $S T / H R$ slope equivalent to single vessel (I), double vessel (II), or triple vessel disease (III), as obtained in selected patients with angina, ${ }^{1}$ are given on the right of the figure. correlation between a reduction in cardiothoracic ratio and the maximal ST/HR slope over a period of six months was found only in one of the 18 patients from the underestimate group who were reexamined $\left(\chi^{2}, \mathrm{p}<0.01\right)$.

Of the 17 patients examined by echocardiography we had adequate images for measurement of left ventricular areas at end diastole in eight. The change in cardiothoracic ratio for these eight patients correlated with the change in the cross sectional end diastolic area of the left ventricle obtained from the apical four chamber view on cross sectional echocardiography $(r=0.79, p<0.02)$.

\section{Discussion}

\section{MAXIMAL ST/HR SLOPE AS INDEX OF \\ MYOCARDIAL ISCHAEMIA}

The maximal ST/HR slope has been proposed as an index of myocardial ischaemia. ${ }^{1}$ In trials of hospital patients presenting with angina in Leeds, it was shown that the slope was $96.5 \%$ successful in detecting significant coronary artery stenosis ${ }^{2}$ (as defined above). These patients were selected; they did not have left ventricular aneurysm, or conditions causing cardiac enlargement-for example valvular heart disease-and they were not examined within six months of myocardial infarction.

In a subsequent trial, patients with aortic valve disease and cardiac enlargement were examined. ${ }^{3}$ In the absence of coronary artery narrowing cardiac enlargement was associated with values of the maximal ST/HR slope equivalent to coronary heart disease. ${ }^{3}$ Left ventricular dilatation and hypertrophy is a reported cause of myocardial ischaemia. ${ }^{4}$

Attempts to reproduce the accuracy found in Leeds have to date been unsuccessful ${ }^{17-19}$; the reason for this is not known. It may be related to the characteristics of the patients studied, use of different techniques, and differences in angiographic interpretation. Other studies of the ST segment and heart rate relation that used different techniques and different angiographic criteria have suggested that this index provides a reliable assessment of the presence and severity of coronary disease in patients with angina. ${ }^{20-22}$ False positive results have been reported in patients with aortic regurgitation ${ }^{23}$ and false negative results in patients with previous myocardial infarction. ${ }^{22} 23$

This evidence suggests that the ability of the maximal ST/HR slope to detect any one cardiac or coronary lesion that leads to myocardial ischaemia must depend on the characteristics of the selected population being studied. Patients early after myocardial infarction could have, in addition to any coronary narrowing, cardiac lesions, such as enlargement or 
scarring, that influence myocardial ischaemia. For instance, left ventricular wall scarring and loss of myocardium may limit myocardial ischaemia whereas cardiac enlargement with a better preserved myocardium may predispose to myocardial ischaemia. ${ }^{4-6}$ The present trial was planned to examine the effects of these factors on the maximal ST/HR slope, which is used as an index of myocardial ischaemia. We used the same techniques in assessing the maximal ST/HR slope that were used in previous studies ${ }^{1112}$ so that we could compare the results of the present trial with those of earlier ones.

FINDINGS OF THE PRESENT STUDY

The present findings suggest that coronary artery narrowing, myocardial scarring, and cardiac enlargement may affect the maximal ST/HR slope after recent myocardial infarction. Myocardial scarring would be associated with left ventricular dilatation, accompanied by an increase in end systolic volume, and reduced wall mobility. The left ventricular score was used as an index of scarring; the finding of an association between high scores and larger left ventricular volumes and a lower ejection fraction (fig 3) confirms that the score was an adequate, though crude, index of scarring. Underestimation of the findings of coronary angiography by the maximal ST/HR slope was found to be significantly associated with the index of myocardial scarring; namely, higher left ventricular scores (fig 2 ), and also with larger left ventricular volumes and a lower ejection fraction (table 1 and fig 4).

It could be argued that two "opposing" areas of myocardial ischaemia could be cancelling out their electrocardiographic manifestations, though there is no evidence for such an effect from experience with the maximal ST/HR slope in patients with angina. ${ }^{1}$ The ST segment elevation associated with aneurysm formation could also be said to mask the presence of ischaemia, though aneurysms may be regarded as mainly representing scar tissue and would be included in the index of scarring used. In the present study many of the patients who had a maximal ST/HR slope of zero had significant narrowing of only the infarct related coronary artery, and this was often associated with poor left ventricular function and in some with aneurysm formation.

One of the principal determinants of prognosis in patients after myocardial infarction is the extent of myocardial damage-a larger infarct being associated with a poorer prognosis. ${ }^{24}$ Though the number of patients in the present study is too small for adequate statistical analysis, the patients in whom the slope underestimated the angiographic extent of coronary artery narrowing had the highest mortality-all three patients who died came from this group. All these findings suggest that scarring may limit myocardial ischaemia as assessed by a functional index, the maximal ST/HR slope.

In this study larger left ventricular volumes were associated with indices of a greater degree of myocardial scarring. The above findings also suggest that scarring may limit myocardial ischaemia. In patients with well preserved left ventricular function - that is less scarring - it may be possible to demonstrate the effect of cardiac enlargement on myocardial ischaemia.

The influence of cardiac enlargement was assessed by correlating individual changes in heart size in the six months after myocardial infarction with changes in the maximal ST/HR slope. The cardiothoracic ratio is an accepted measure of change in heart size in an individual, ${ }^{9}$ though it is not accurate for comparisons between patients. In this study correlation with echocardiographic measurements showed that changes in cardiothoracic ratio reflected alterations in left ventricular dimensions. Examination of these indices showed that there was evidence that ventricular size affected the maximal ST/HR slope.

In the group in which the slope overestimated left ventricular damage (that is those with the best preserved left ventricular function) most patients demonstrated a reduction in cardiothoracic ratio which was associated with a reduction in the maximal ST/HR slope. The reduction in the slope exceeded the $95 \%$ tolerance limits of obtaining the slope ${ }^{1}$ and for some patients involved a shift from the range of double vessel disease to single vessel disease.

In this study we obtained values of the slope that were intermediate between the categories of single and double vessel disease and double and triple vessel disease. It is our experience that such values are often obtained when cardiac enlargement has been evident. $^{3}$

Even in the face of factors such as scarring and cardiac enlargement that influence myocardial ischaemia there was still a tendency for the assessment of myocardial ischaemia by the maximal ST/HR slope to agree with assessment by coronary angiography (fig 1). Where the slope agreed with the angiographic findings coronary artery narrowing may not have been the principal influence on myocardial ischaemia since scarring and cardiac enlargement could also have been influencing the results.

\section{IMPLICATIONS}

The above findings suggest that, in addition to being dependent on the severity of coronary artery disease, myocardial ischaemia early after myocardial infarction may be limited by scarring and may be increased by cardiac enlargement. This implies that in individual patients exercise induced myocardial 
ischaemia after recent myocardial infarction is determined by at least these three factors; however, the relative contribution of each is not known.

Cardiac enlargement in patients with myocardial infarction is also related to the degree of scarring, ${ }^{13}$ and so differs from the enlargement seen with aortic valve disease. The present findings suggest that scarring may limit myocardial ischaemia attributable to coronary artery narrowing, and scarring also is likely to limit the influence of cardiac enlargement on the ischaemic process. This is evident from the group in which the slope underestimated the extent of coronary artery narrowing and who also had the largest left ventricular volumes (table). Animal studies have shown that a relative increase in left ventricular volume occurs even with small infarctions. ${ }^{25}$ Where the effect of scarring is not great it should be possible to detect, using an index of myocardial ischaemia, the contribution of cardiac enlargement to the ischaemic process. This is supported by the findings from the patients in whom the slope overestimated the severity of coronary artery narrowing; this group had the best preserved left ventricular function.

Other possible explanations for the overestimation of coronary artery narrowing by the slope that was seen in some patients include drug treatment, distal coronary artery disease, and haematological abnormalities. An increase in the maximal ST/HR slope was seen when vasodilating drugs were used in patients with stable angina ${ }^{15}{ }^{16}$; however, only two of the patients in the overestimate group were on such treatment. It is possible that distal coronary disease may be contributing to this "extra ischaemia"; trials of the use of the maximal ST/HR slope in patients with stable angina did not assess this aspect. ${ }^{1}$ Abnormalities of platelet morphology have been reported in patients after acute myocardial infarction, ${ }^{26}$ though further study is required to find out whether these changes contribute to ischaemia in the weeks after myocardial infarction.

The present findings may help to explain the reported discrepancy in the published reports regarding the value of exercise electrocardiography after infarction in detecting "multivessel disease". 2728 Although the index of myocardial ischaemia used in such trials is open to criticism, ${ }^{1}$ the discrepancy in results may also be due to influences other than coronary artery disease on myocardial ischaemia.

\section{CONCLUSION}

This study involving blind comparison of the maximal ST/HR slope with angiocardiography was aimed at testing the accuracy of the slope as an index of myocardial ischaemia early after myocardial infarction. The slope has been shown to be sensitive to the modifying influences of myocardial scarring and cardiac enlargement upon myocardial ischaemia soon after infarction. Exercise induced ischaemia early after myocardial infarction is influenced by myocardial scarring, cardiac enlargement, and significant narrowing of the coronary artery. Because ischaemia early after myocardial infarction is multifactorial, unlike ischaemia in patients with stable angina, the slope cannot be expected to detect the extent of significant coronary artery narrowing.

We thank Drs A F Mackintosh and G Williams for permission to study their patients.

\section{References}

1 Linden RJ, Mary DASG. Limitations and reliability of exercise electrocardiography tests in coronary heart disease. Cardiovasc Res 1982;16:675-710.

2 Mary DASG, Silverton NP, Boyle RM, Stoker JB, Smith DR, Linden RJ. The ST/HR slope as an index of ischaemic heart disease [Abstract]. Circulation 1985;72 (suppl III):163.

3 Bishop N, Boyle R, Watson DA, Mary DASG, Linden $R J$. The contribution of cardiac enlargement to myocardial ischaemia-assessment using the maximal ST/HR slope in patients before and after aortic valve surgery [Abstract]. Clin $S_{c i}$ 1985;69 (suppl 12):55-6P.

4 Hoffman JIE. Why is myocardial ischaemia so commonly subendocardial? Clin Sci 1981;61:657-62.

5 Ellestad MH. Stress testing. Principles and practice. Philadelphia: Davis, 1981:321-3.

6 Castellanet MJ, Greenberg PS, Ellestad MH. Comparison of ST segment changes on exercise testing with angiographic findings in patients with prior myocardial infarction. Am J Cardiol 1978;42:29-35.

7 Cohn PF, Herman MV, Gorlin R. Profiles in coronary artery disease. In: Grossman W, ed. Cardiac catheterization and angiography. 2nd ed. Philadelphia: Lea and Febiger, 1980:325-35.

8 Baron MG. Radiological and angiographic examination of the heart. In: Braunwald E, ed. Heart disease. Philadelphia: WB Saunders, 1980:147-97.

9 Chen JTT. The chest roentgenogram. In: Hurst JW, ed. The heart. New York: McGraw Hill, 1982:278.

10 Feigenbaum H. Echocardiography. 3rd ed. Philadelphia: Lea and Febiger, 1981:149-53.

11 Elamin MS, Boyle R, Kardash MM, et al. Accurate detection of coronary heart disease by new exercise test. Br Heart $J$ 1982;48:311-20.

12 Elamin MS, Mary DASG, Smith DR, Linden RJ. Prediction of severity of coronary artery disease using slope of submaximal ST segment/heart rate relationship. Cardiovasc Res 1980;14:681-91.

13 Lamas GA, Pfeffer MA. Increased left ventricular volume following myocardial infarction in man. Am Heart $J$ 1986;111:30-5.

14 Kennedy JW, Trenholme SE, Kasser IS. Left ventric- 
ular volume and mass from single plane cineangiocardiograms: a comparison of antero-posterior and right anterior oblique methods. Am Heart $J$ 1970;80:343-52.

15 Bishop N, Hart G, Elamin MS, et al. Assessment of the effect of nifedipine on myocardial ischaemia by using the ST segment/heart rate slope. Clin Sci 1986; 70:601-9.

16 Bishop N, Linden RJ, Mary DASG, Stoker JB. The effect of vasodilator drugs on myocardial ischaemia in stable angina using the maximal ST/HR slope [Abstract]. Clin Sci 1985;70(suppl 13):8P.

17 Quyyumi AA, Raphael MJ, Wright C, Bealing L, Fox $\mathrm{KM}$. Inability of the $S T$ segment/heart rate slope to predict accurately the severity of coronary artery disease. Br Heart J 1984;51:395-8.

18 Balcon R, Brooks N, Layton C. Correlation of heart rate/ST slope and coronary angiographic findings. Br Heart J 1984;52:304-7.

19 Thwaites BC, Quyyumi AA, Raphael MJ, Canepa-Anson R, Fox KM. Comparison of the ST/heart rate slope with the modified Bruce exercise test in the detection of coronary artery disease. $\mathrm{Am} \mathrm{J}$ Cardiol 1986;57:554-6.

20 Okin PM, Kligfield P, Ameisen O, Goldberg HL, Borer JS. Improved accuracy of the exercise electrocardiogram: identification of three-vessel coronary disease in stable angina pectoris by analysis of peak rate-related changes in ST segments. Am J Cardiol 1985;55:271-6.

21 Berenyi I, Hajduczki IS, Boszormenyi I. Quantitative evaluation of exercise-induced ST segment depression for estimation of degree of coronary artery disease. Eur Heart $J$ 1984;5:289-94.

22 Finkelhor RS, Newhouse KE, Vrobel TR, Miron SD, Bahler RC. The ST segment/heart rate slope as a predictor of coronary artery disease: comparison with quantitative thallium imaging and conventional ST segment criteria. Am Heart J 1986;112:296-304.

23 Ameisen O, Okin PM, Devereux RB, et al. Predictive value and limitations of the $S T / H R$ slope. Br Heart $J$ 1985;53:547-51.

24 Rackley CE, Russell RO, Mantle JA, Rogers WJ, Papapietro SE. Modern approach to myocardial infarction: determination of prognosis and therapy. Am Heart J 1981;101:75-85.

25 Fletcher PJ, Pfeffer JM, Pfeffer MA, Braunwald E. Left ventricular diastolic pressure-volume relations in rats with healed myocardial infarction. Circ Res 1981;49:618-26.

26 Martin JF, Plumb J, Kilbey RS, Kishk YT. Changes in volume and density of platelets in myocardial infarction. Br Med J 1983;287:456-8.

27 Akhras F, Upward J, Stott R, Jackson G. Early exercise testing and coronary angiography after uncomplicated myocardial infarction. $B r \operatorname{Med} J$ 1982;284: 1293-4.

28 Peart I, Seth L, Albers C, Odemuyiwa O, Hall RJC. Post-infarction exercise testing in patients under 55 years: relation between ischaemic abnormalities and the extent of coronary artery disease. $\mathrm{Br}$ Heart $J$ 1986;55:67-74. 